\section{Fatores de risco para mortalidade infantil em município do Nordeste do Brasil: linkage entre bancos de dados de nascidos vivos e óbitos infantis - 2000 a 2002}

\author{
Risk factors for infant mortality in a \\ city of northeastern Brazil: linkage \\ between live birth and infant death \\ databases - 2000 to 2002
}

\author{
Cristiana Ferreira da Silva ${ }^{1}$ \\ Álvaro Jorge Madeiro Leite ${ }^{2}$ \\ Nádia Maria Girão Saraiva de Almeida ${ }^{3}$ \\ Rogério Costa Gondim ${ }^{4}$ \\ 'Secretaria de Saúde da Prefeitura Municipal de Maracanaú, CE, Brasil \\ ${ }^{2}$ Professor adjunto de Pediatria do Departamento de Saúde Materno Infantil. \\ Faculdade de Medicina. Universidade Federal do Ceará. Brasil. \\ ${ }^{3}$ Doutora em Saúde Pública. Escola de Saúde Pública do Ceará. Brasil. \\ ${ }^{4}$ Secretaria da Saúde do Estado do Ceará, Brasil.
}

Endereço para correspondência: Cristiana Ferreira da Silva. Secretaria de Saúde da Prefeitura Municipal de Maracanaú. Rua Pedro de Queiroz, 758. Fortaleza, CE, Brasil. CEP: 60.450-220. E-mail: cristianaferreirasilva@yahoo.com.br

\section{Resumo}

Objetivo: Analisar fatores de risco para a morte de crianças menores de um ano de idade, do município de Maracanaú, Estado do Ceará, entre os anos de 2000 e 2002, através de linkage das informações do Sistema de Informação sobre Nascidos Vivos e Sistema de Informação sobre Mortalidade. Métodos: Coorte retrospectiva de 11.127 nascimentos vivos com declaração de nascido vivo preenchida, ocorridos entre $01 / 01 / 2000$ e 31/12/2002 e 119 óbitos infantis relacionados a essa coorte de nascidos vivos, com declaração de óbito ou instrumento de investigação de óbito infantil preenchidos, ocorridos entre 01/01/ 2000 e $31 / 12 / 2003$. Os fatores de risco para a mortalidade infantil foram estimados utilizando-se as análises bivariada e multivariada através da regressão logística. Resultados: Foram encontradas odds ratio (OR) estatisticamente significativas para nascidos vivos com baixo peso ao nascer $\left(\mathrm{OR}=3,16\right.$; $\left._{95 \%} 1,58-6,35\right)$, nascidos vivos prematuros $\left(\mathrm{OR}=2,70\right.$; $\left.\mathrm{IC}_{95 \%} 1,25-5,86\right)$, nascidos vivos de mães com consultas prénatal igual ou menores a $6\left(\mathrm{OR}=2,05 ; \mathrm{IC}_{95 \%}\right.$ $1,15-3,64)$ e nascidos vivos cujo escore de Apgar no primeiro $\left(\mathrm{OR}=4,40 \mathrm{IC}_{95 \%} 2,48\right.$ $7,81)$ e quinto $\left(\mathrm{OR}=5,5 ; \mathrm{IC}_{95 \%} 2,75-11,20\right)$ minutos de vida foram inferiores a sete. Conclusões: Esse estudo possibilitou o uso das bases de dados de nascimentos e óbitos, sugerindo a vigilância contínua e oferta de assistência adequada ao subgrupo de recém-nascidos com maior risco de morte e a garantia de assistência pré-natal de qualidade.

Palavras-chave: Mortalidade infantil. Sistemas de informação. Registros. 
Abstract

Objective: to analyze risk factors for the death of children at one year of age in the town of Maracanaú, in the State of Ceará, between the year 2000 and 2002, through the linkage of information of the System of Information on Live Births and the Mortality System of Information. Methods: retrospective cohort of 11,127 live births with certificate of born-alive filled out and occurred between 01/01/2000 and 31/01/ 2002 and 119 infant deaths linked to this cohort of born-alive, with certificate of death or investigation instrument of infant death filled out and occurred between 01/ $01 / 2000$ and $31 / 01 / 2003$. The risk factors for the infant death were estimated utilizing the bi-varied and multi-varied analysis through the logistic regression. Results: significant Odds Ratio (OR) were found among born-alive with low weight when coming to life (OR=3.16; $\mathrm{IC}_{95 \%}$ 1.58-6.35), premature born-alive $\left(\mathrm{OR}=2.70 ; \mathrm{IC}_{95 \%} 1.25\right.$ 5.86), born-alive from mothers with prenatal appointment the same as or lower than $6\left(\mathrm{OR}=2.05\right.$; $\left.\mathrm{IC}_{95 \%} 1.15-3.64\right)$ and bornalive whose Apgar score in the first $\left(\mathrm{OR}=4.40 ; \mathrm{IC}_{95 \%} 2.48-7.81\right)$ and the fifth $\left(\mathrm{OR}=5.5 ; \mathrm{IC}_{95 \%} 2.75-11.20\right)$ minutes of life were lower than seven. Conclusions: this study made it possible the use of data bases of birth and death, suggesting the continuous vigilance and offer of adequate assistance to the subgroup of newly-born with a higher risk of death and the guarantee of pre-natal assistance of quality.

Keywords: Infant Mortality. Information System. Records.

\section{Introdução}

O coeficiente de mortalidade infantil reflete as condições sensíveis da população, sendo considerada por inúmeros autores e organismos internacionais como um indicador clássico e consagrado do nível de vida $^{1-7}$. É uma medida que reflete as condições de vida da população, associando o nível de vida, por exemplo, a alimentação, moradia e acesso ao conhecimento médico, como determinantes de sobrevivência no primeiro ano de vida ${ }^{8}$. Nesse contexto, associam-se às causas biológicas da morte infantil aquelas de ordem social, econômica e ambiental. Os óbitos infantis estão mais propensos a determinantes sociais do que os ocorridos na idade adulta, devido ao organismo infantil ser um complexo psicobiológico em formação, com a capacidade de defesa das agressões externas naturalmente reduzida, sendo freqüentemente exposto a inúmeras doenças e complicações que potencializam o risco da morte infantil ${ }^{9}$.

Os fatores determinantes da mortalidade infantil e da sobrevivência infantil são alvo de preocupação entre a comunidade científica brasileira e internacional. Organismos e instituições governamentais e não-governamentais reconhecem como desafiadora a luta pela manutenção de taxas cada vez menores desse indicador ${ }^{10}$.

O estudo dos fatores de risco dos óbitos entre crianças menores de um ano possibilita a elucidação da rede de eventos determinantes, a identificação de grupos expostos, bem como das necessidades de saúde de subgrupos populacionais, permitindo a programação de intervenções voltadas à redução dos óbitos infantis ${ }^{11}$.

$\mathrm{O}$ interesse em relacionar registros em diferentes bases de dados vem aumentando progressivamente de acordo com a crescente disponibilidade de grandes bancos de dados informatizados na área da saúde, assim como pelo avanço tecnológico na área da computação, tornando disponíveis aplicativos e máquinas cada vez mais eficazes ${ }^{12,13}$. 
"Linkage" significa literalmente ligação. Consiste na ligação de dois ou mais bancos de dados independentes, mas que tem a característica de possuírem variáveis em comum. Assim é possível identificar indivíduos ou registros que fazem parte dos dois bancos de dados. A ligação dos dois bancos permite o estabelecimento de um banco de dados único, contendo variáveis dos dois bancos de dados ${ }^{13}$.

A principal vantagem da utilização dessa técnica ou procedimento é o baixo custo, visto que os dados encontram-se registrados, aguardando apenas uma adequada análise para definição de priorida$\operatorname{des}^{14}$. Pode-se dessa forma explorar as informações registradas nos Sistemas de Informação sobre Nascidos Vivos (SINASC) e sobre Mortalidade (SIM), objetivando a identificação dos riscos e o planejamento da atenção à saúde materno-infantil. $\mathrm{O}$ procedimento de "linkage" torna viável o estudo da mortalidade infantil em coortes de nascidos vivos de base populacional; a estimativa direta da probabilidade de morte infantil; a estimativa de morte em recém-nascidos, segundo variáveis presentes na declaração de nascido vivo (DN); o estabelecimento de associação entre as variáveis independentes da DN e a mortalidade infantil; e finalmente, permite a plena utilização de dados oficiais secundários, disponibilizados no SINASC e SIM em estudos analíticos longitudinais ${ }^{15,16}$.

Este estudo objetivou identificar os fatores de risco para a mortalidade infantil a partir de uma coorte de nascidos vivos, utilizando como variáveis preditoras aquelas contidas na declaração de nascido vivo.

\section{Métodos}

Coorte retrospectiva de nascidos vivos. Foi realizado com os dados do município de Maracanaú, localizado na região metropolitana de Fortaleza, CE, Brasil. A população constituiu-se de 11.127 nascimentos vivos com DN preenchida ocorridos entre 01 de janeiro de 2000 e 31 de dezembro de 2002, filhos de mães residentes em
Maracanaú e de 119 óbitos infantis relacionados a essa coorte de nascidos vivos, com declaração de óbito (DO) ou instrumento de investigação de óbito infantil preenchido, ocorridos entre 01 de janeiro de 2000 e 31 de dezembro de 2003.

Os dados foram provenientes do SINASC, SIM e do instrumento de investigação de óbito infantil (ficha de autópsia verbal ou investigação de óbito em menores de um ano) realizado pelas equipes do Programa Saúde da Família (PSF), cedidos pela Secretaria de Saúde de Maracanaú e pela Secretaria de Saúde do Estado do Ceará. O procedimento de linkage determinístico (busca manual) foi utilizado entre os bancos de dados de óbitos e nascimentos a fim de identificar os nascidos vivos que evoluíram para óbito infantil. Os campos nome da mãe, data do nascimento e sexo foram utilizados para a verificação de linkage e emparelhamento da DN com sua respectiva DO. Conseguiu-se a ligação de 119 óbitos infantis às suas respectivas DN. O procedimento de linkage não conseguiu identificar, dos 211 óbitos registrados no SIM e resgatados pelas investigações de óbito infantil, a DN de 92 registros de mortes em menores de um ano de idade (nascidos vivos em que não houve provavelmente emissão da DN) e 72 registros de nascimentos foram excluídos da população de estudo por se encontrarem em duplicidade nos bancos de dados analisados.

A variável dependente do estudo foi a ocorrência de morte antes de completar o primeiro ano de vida e as variáveis de exposição: sexo (masculino e feminino), peso ao nascer (menor que 2.500 gramas e igual ou superior a 2.500 gramas), índice de Apgar no primeiro e quinto minutos de vida (menor que 7 e igual ou superior a 7), tipo de gravidez (única e múltipla), duração da gestação (menor que 37 semanas, 37 a 41 semanas e igual ou maior que 42 semanas), número de consultas pré-natal (nenhuma, 1 a 6 consultas e 7 ou mais), tipo de parto (normal e operatório), grau de instrução da mãe (nenhum, 1 a 7 anos de es- 
tudo, 8 a 11 anos de estudo e 12 ou mais anos de estudo), idade da mãe (menos de 20 anos, 20-34 anos e igual ou maior que 35 anos), paridade (primípara e multípara), local de ocorrência do nascimento (hospital, domicílio, outros), categoria econômica do estabelecimento de saúde do nascimento (público e privado conveniado ao Sistema Único de Saúde - SUS).

As análises estatísticas foram realizadas utilizando-se os softwares Epi-info versão 6.04 e Stata 7.0. As análises bivariada e multivariada testaram a associação dos vários fatores preditores com a mortalidade infantil. As medidas de associação empregadas foram o risco relativo e Odds Ratio $(O R)$, assumindo-se nesse estudo intervalos de confiança em nível de $95 \%$ e a regressão logística foi utilizada como procedimento para o ajustamento de efeitos de confusão. Para a seleção do modelo final, foram incluídas todas as variáveis com valor de $\mathrm{p}<0,25$ na análise bivariada, tendo sido incluída no modelo a variável escolaridade materna, por sua importância do ponto de vista clínico-epidemiológico na determinação da mortalidade infantil tal como descrita na literatura revisada. Foram considerados como potenciais fatores de confusão as variáveis selecionadas que alteraram em 10\% (para mais ou para menos) os valores da medida de $O R$. O componente de erro foi mensurado através do teste de ajustamento (godness of fit test), encontrando-se Qui-quadrado HosmerLemeshow=5,28 e valor de $\mathrm{p}=0,6253$.

Os referenciais da bioética, preconizados na resolução n. ${ }^{\circ}$ 196/96 do Conselho Nacional de Saúde (CNS) foram considerados nesse estudo, tendo sido obtido o consentimento do então Secretário de Saúde e Ação Social do município de Maracanaú e a aprovação do Comitê de Ética em Pesquisa do Complexo Hospitalar da Universidade Federal do Ceará.

\section{Resultados}

Do total de 119 óbitos infantis analisados nesse estudo, 75 (63,0\%) ocorreram no período neonatal - antes de completarem 28 dias de vida - e 44 (37\%), no período pós neonatal - de 28 dias a um ano de idade. Dos óbitos neonatais, 56\%(42/75) ocorreram no período precoce - até 6 dias de vida, e $44 \%(33 / 75)$ referiram-se a mortalidade neonatal tardia - de 7 até 28 dias de vida. O percentual de óbito nas primeiras 24 horas foi de $18,6 \%$ (14/75). A probabilidade de morrer antes de completar o primeiro ano de vida, utilizando-se como numerador o número de óbitos relacionados (119/11127), foi de 10,7 para cada mil nascidos vivos, sendo 6,7 por mil no período neonatal, e 3,9 por mil no período pósneonatal, mas alcançando taxa de 19,0 por mil nascidos vivos, utilizando-se como numerador o número real de óbitos (211/ 11127) registrados no SIM e nas investigações de óbito infantil. As diferenças entre as taxas encontradas nesse estudo ocorreram devido às exclusões de óbitos infantis e nascimentos. Acrescentamos que o subregistro de óbitos ocasionou uma subestimativa do coeficiente de mortalidade infantil.

Em relação aos nascimentos, houve predomínio de recém-nascidos do sexo masculino $(5.731 / 51,7 \%)$. O peso médio dos nascimentos foi de $3.222 \mathrm{~g}$ (desvio padrão = 541g). A incidência de recém-nascidos com baixo peso (menor $2.500 \mathrm{~g}$ ) e muito baixo peso ao nascer (menos de $1.500 \mathrm{~g}$ ) entre todos os nascidos vivos foi de $7,4 \%$ e $0,9 \%$ respectivamente. $O$ índice de Apgar apresentou valores inferiores a sete em $6,4 \%$ dos nascidos vivos no primeiro minuto de vida e em $1,4 \%$ no quinto minuto de vida. As gestações a termo (37-41 semanas) corresponderam a $95,1 \%$ do total dos nascimentos vivos, os nascidos vivos pré-termo (até 36 semanas) representaram $4,2 \%$ dos recém-nascidos, enquanto os nascimentos pós-termo alcançaram $0,7 \%$ do total de DN analisadas. Do total de nascimentos, 98,5\% corresponderam a gestações únicas e 1,5\% foram referentes a gestações múltiplas, das quais $15(0,1 \%)$ foram de gestações triplas. O percentual de nascimentos cujas mães realizaram de 4 a 
6 consultas de pré-natal foi de 50,8\%; 39,9\% realizaram de 7 a mais consultas; cerca de $7 \%$ tiveram de 1 a 3 consultas e 1,7\% não realizaram consulta de assistência ao prénatal. De acordo com o número de filhos tidos anteriormente, 30,9\% foram referentes a nascidos vivos de mães que não tiveram outros filhos e $69,1 \%$ eram de multíparas (de um ou mais filhos tidos anteriormente). O parto vaginal predominou, alcançando 64,7\% do total de nascimentos e a proporção de cesáreas foi da ordem de $35,3 \%$. Dentre todos os partos operatórios, $28,7 \%$ ocorreram em nascidos vivos de mães primíparas e $71,3 \%$ foram realizados em multíparas. O percentual de parto vaginal entre multíparas foi de $68,1 \%$ e entre as primíparas, de $31,9 \%$. A média da idade materna foi de 24,3 anos (desvio padrão =6,266), com valor mínimo de 12 anos e máximo de 49 anos de idade. Observouse que $24,7 \%$ dos nascidos vivos eram de mães adolescentes (com idade inferior a 20 anos) e 7,3\% possuíam idade de 35 anos ou mais. A distribuição dos nascidos vivos em relação ao grau de instrução da mãe indicou que 58,1\% (6.181) dos nascimentos pertenciam à categoria de 1 a 7 anos de estudo, 29,5\% (3.139) tinham de 8 a 11 anos de estudo, 6,1\% (652) encontravamse na faixa de 12 ou mais anos de estudo e $6,2 \%$ (660) dos nascimentos tinham mães com nenhum nível de instrução. Cerca de 99,6\% dos nascimentos dessa coorte foram hospitalares e apenas $0,3 \%$ ocorreram no domicílio. Ressaltamos que 7 nascidos vivos tiveram em sua DN registro de nascimento em outros locais (via pública) e outros estabelecimentos de saúde que não hospitalares. Dos nascimentos analisados, em 766 DN não havia registro do código do estabelecimento de saúde $(6,9 \%$ do total). Do total de registros com preenchimento do referido campo, 43,3\% (4.482) ocorreram em hospital público e $56,7 \%$ (5.879) em estabelecimento privado conveniado com o SUS.

A Tabela 1 apresenta o coeficiente de mortalidade infantil e os resultados da análise bivariada para os óbitos infantis de acordo com as variáveis contidas na DN. Foram identificadas associações estatisticamente significativas entre mortalidade infantil e as variáveis independentes para os seguintes subgrupos de nascidos vivos: os nascidos vivos com baixo peso ao nascer (menor 2500g) apresentaram risco de morte elevado quando comparados aos nascidos vivos com peso igual ou superior a $2.500 \mathrm{~g}\left(\mathrm{RR}=10,51 ; \mathrm{IC}_{95 \%} 7,37-15,00\right)$; os nascidos vivos com índice de Apgar inferior a sete no primeiro $\left(\mathrm{RR}=9,94 ; \mathrm{IC}_{95 \%} 6,89\right.$ $14,34)$ e quinto $\left(\mathrm{RR}=18,55 ; \mathrm{IC}_{95 \%} 12,15\right.$ $28,31)$ minutos de vida; os nascidos vivos prematuros apresentaram risco de morte superior ao dos nascidos vivos de termo $\left(\mathrm{RR}=12,92 ; \mathrm{IC}_{95 \%} 8,91-18,74\right)$; os nascidos vivos de gravidez múltipla apresentaram um risco de morte antes de completar um ano de vida 4,63 vezes superior quando comparados aos nascidos vivos de gravidez única; nascidos vivos de mães que não tiveram nenhuma consulta de pré-natal $\left(\mathrm{RR}=8,66\right.$; $\left._{95 \%} 3,91-19,18\right)$ e nascidos vivos de mães que realizaram de uma a seis consultas pré-natal $\left(\mathrm{RR}=2,60 ; \mathrm{IC}_{95 \%} 1,63\right.$ $4,15)$, em relação aos nascidos vivos de mães que tiveram sete ou mais consultas de pré-natal; os nascidos vivos de mães com idade igual ou superior a 35 anos apresentaram risco para morte 2,3 vezes superior quando comparados aos nascidos vivos de mães entre 20 e 34 anos de idade; os nascidos vivos de partos domiciliares apresentaram risco de morte 5,92 vezes superior quando comparados aos nascidos vivos de partos hospitalares. As variáveis independentes: sexo do recém-nascido, paridade, tipo de parto, escolaridade da mãe em anos de estudo e categoria econômica do estabelecimento de saúde não mostraram associação estatisticamente significativa com a mortalidade infantil.

A Tabela 2 mostra o resultado da análise multivariada. Constituíram-se, de acordo com o modelo construído, fatores de risco independentes para o óbito infantil as variáveis baixo peso ao nascer, duração da gestação, escore de Apgar inferior a sete no primeiro e quinto minutos de vida e 
Tabela 1 - Resultados da análise bivariada, coeficiente de mortalidade infantil (CMI), Risco Relativo para óbito infantil segundo variáveis da Declaração de Nascido Vivo. Maracanaú, Ce, 2000 a 2002.

Table 1 - Results of bivariate analysis, infant mortality rates (CIM), relative risk for infant deaths, according to variables of Live Born Certificates. Maracanaú, CE, from 2000 to 2002.

\begin{tabular}{|c|c|c|c|c|c|c|}
\hline Variáveis & CMI & $\begin{array}{r}\text { Óbitos } \\
\mathrm{N}=119\end{array}$ & $\begin{array}{c}\text { Sobreviventes } \\
\mathrm{N}=11008\end{array}$ & $\begin{array}{l}\text { RR não } \\
\text { ajustado }\end{array}$ & IC95\% & $\mathrm{p}$ \\
\hline \multicolumn{7}{|l|}{ Sexo } \\
\hline Feminino & 11,0 & 59 & 5.297 & 1,0 & & \\
\hline Masculino & 10,4 & 60 & 5.671 & 0,95 & $0,66-1,36$ & 0,851 \\
\hline \multicolumn{7}{|c|}{ Peso ao nascer (g) } \\
\hline$\geq 2500$ & 6,2 & 64 & 10.203 & 1,0 & & \\
\hline$<2500$ & 65,5 & 54 & 770 & 10,51 & $7,37-15,00$ & $<0,001$ \\
\hline \multicolumn{7}{|l|}{ Apgar 1 minuto } \\
\hline$\geq 7$ & 6,6 & 68 & 10.159 & 1,0 & & \\
\hline$<7$ & 66,0 & 46 & 650 & 9,94 & $6,89-14,34$ & $<0,001$ \\
\hline \multicolumn{7}{|l|}{ Apgar 5 minuto } \\
\hline$\geq 7$ & 8,2 & 88 & 10.522 & 1,0 & & \\
\hline$<7$ & 181,8 & 24 & 132 & 18,55 & $12,15-28,31$ & $<0,001$ \\
\hline \multicolumn{7}{|c|}{ Duração da gestação (semanas) } \\
\hline $37-41$ & 6,8 & 72 & 10.365 & 1,0 & & \\
\hline$<37$ & 89,1 & 41 & 419 & 12,92 & $8,91-18,74$ & $<0,001$ \\
\hline$\geq 42$ & - & 0 & 80 & - & - & - \\
\hline \multicolumn{7}{|l|}{ Tipo de gravidez } \\
\hline Única & 10,1 & 111 & 10.801 & 1,0 & & \\
\hline Múltipla & 47,0 & 08 & 162 & 4,63 & $2,29-9,33$ & $<0,001$ \\
\hline \multicolumn{7}{|c|}{ Consultas pré-natal } \\
\hline Mais de 6 & 5,1 & 22 & 4.288 & 1,0 & & \\
\hline Nenhuma & 44,1 & 08 & 173 & 8,66 & $3,91-19,18$ & $<0,001$ \\
\hline Até 6 & 13,2 & 84 & 6.240 & 2,60 & $1,63-4,15$ & $<0,001$ \\
\hline \multicolumn{7}{|l|}{ Paridade } \\
\hline Multípara & 10,8 & 74 & 6.726 & 1,0 & & \\
\hline Primípara & 8,8 & 27 & 3.008 & 0,82 & $0,53-1,27$ & 0,427 \\
\hline \multicolumn{7}{|l|}{ Tipo de parto } \\
\hline Normal & 11,0 & 79 & 7.100 & 1,0 & & \\
\hline Operatório & 10,2 & 40 & 3.870 & 0,93 & $0,64-1,36$ & 0,778 \\
\hline \multicolumn{7}{|c|}{ Anos de estudo/mãe } \\
\hline$\geq 12$ & 10,7 & 07 & 645 & 1,0 & & \\
\hline Nenhum & 16,6 & 11 & 649 & 1,55 & $0,61-3,98$ & 0,492 \\
\hline $1-7$ & 11,1 & 69 & 6.112 & 1,04 & $0,48-2,25$ & 0,922 \\
\hline 8-11 anos & 7,9 & 25 & 3.114 & 0,74 & $0,32-1,71$ & 0,639 \\
\hline \multicolumn{7}{|c|}{ Idade materna (anos) } \\
\hline 20-34 & 9,1 & 69 & 7.483 & 1,0 & & \\
\hline$<20$ & 12,0 & 33 & 2.714 & 1,31 & $0,87-1,99$ & 0,233 \\
\hline$\geq 35$ & 21,0 & 17 & 791 & 2,30 & $1,36-3,90$ & 0,002 \\
\hline \multicolumn{7}{|c|}{ Local de nascimento } \\
\hline Hospital & 10,5 & 117 & 10.971 & 1,0 & & \\
\hline Domicílio & 62,5 & 02 & 30 & 5,92 & $1,53-22,94$ & $0,045^{*}$ \\
\hline Outros & & 00 & 07 & - & - & - \\
\hline \multicolumn{7}{|l|}{ Estabelecimento } \\
\hline Privado/SUS & 8,5 & 50 & 5.829 & 1,0 & & \\
\hline Público & 12,0 & 54 & 4.428 & 1,42 & $0,97-2,08$ & 0,090 \\
\hline
\end{tabular}

* Teste exato de Fisher; Fisher exact test 
número igual ou inferior a seis consultas de pré-natal. As variáveis categoria econômica do hospital de nascimento, idade materna e a escolaridade da mãe permaneceram no modelo final como variáveis de controle. As variáveis sexo do recémnascido, tipo de gravidez e local de nascimento não apresentaram associação estatisticamente significativa e não atuaram como fatores de confusão, o que justificou a exclusão do modelo. Para a seleção do modelo final foram incluídas todas as variáveis com $\mathrm{p}<0,25$ na análise bivariada, tendo sido incluída no modelo a variável escolaridade materna, por sua importância do ponto de vista clínico-epidemiológico na determinação da mortalidade infantil, tal como descrita na literatura revisada.

Os nascidos vivos com baixo peso ao nascer apresentaram um risco 3 vezes maior de morte antes de completar o primeiro ano de vida quando comparados aos de peso igual ou superior a $2.500 \mathrm{~g}(\mathrm{OR}=$ 3,16 ; $\left.\mathrm{IC}_{95 \%} 1,58-6,35\right)$; os nascidos vivos prematuros tiveram um risco de morte 2,7 maior do que os de termo (OR = 2,70; $\mathrm{IC}_{95 \%}$ $1,25-5,86)$; nascidos vivos de mães com o número de consultas pré-natal igual ou menores a $6\left(\mathrm{OR}=2,05 ; \mathrm{IC}_{95 \%} 1,15-3,64\right) \mathrm{e}$ nascidos vivos cujo escore de Apgar no primeiro (OR $\left.=4,40 ; \mathrm{IC}_{95 \%} 2,48-7,81\right)$ e quin- to $\left(\mathrm{OR}=5,5 ; \mathrm{IC}_{95 \%} 2,75-11,20\right)$ minutos de vida foram inferiores a sete.

\section{Discussão}

A oportunidade de utilizar e examinar as informações sobre nascimentos e óbitos infantis do SINASC, SIM e das investigações de óbito infantil favorece novas possibilidades na identificação de fatores determinantes da mortalidade infantil, apesar de dificuldades na ligação dos bancos de dados e da conseqüente sub-enumeração dos nascidos vivos no SINASC, dos óbitos e conseqüentemente da taxa de mortalidade infantil na coorte estudada.

Possíveis falha de emissão e preenchimento da declaração de nascido vivo e de óbito infantil foram descritas em outros estudos $^{13,14,17,18}$, impossibilitando a ligação de óbitos infantis registrados no SIM e nas investigações de óbito infantil. Apesar das restrições metodológicas inerentes ao uso de dados secundários provenientes das DN, DO e investigação de óbito infantil, o procedimento de "linkage" mostrou-se viável do ponto de vista operacional.

O predomínio de óbitos infantis no período neonatal encontrado nessa coorte está em concordância com outros estudos $^{8,15,19-21}$. Vários fatores podem ter contribuído para o aumento relativo da mor-

Tabela 2 - Resultados da análise multivariada da mortalidade infantil segundo variáveis da Declaração de Nascido Vivo. Maracanaú, CE, 2000 a 2002.

Table 2 - Results of multivariate analysis of infant mortality according to variables of Live Born Certificates. Maracanaú, CE, from 2000 to 2002.

\begin{tabular}{llll}
\hline Variáveis & Odds Ratio & $\mathrm{IC}_{95 \%}$ & $\mathrm{p}$ \\
\hline $\begin{array}{l}\text { Peso ao nascer } \\
\quad(1=\text { baixo peso; } 0=\text { peso normal })\end{array}$ & 3,16 & $1,58-6,35$ & 0,001 \\
$\begin{array}{l}\text { Duração da gestação } \\
\quad(1=<37 \text { sem.; } 0=\geq 37 \text { sem. })\end{array}$ & 2,70 & $1,25-5,86$ & 0,012 \\
$\begin{array}{l}\text { Apgar } 1 \text { minuto } \\
\quad(1=<7 ; 0=\geq 7)\end{array}$ & 4,40 & $2,48-7,81$ & $<0,001$ \\
$\begin{array}{l}\text { Apgar } 5 \text { minuto } \\
\quad(1=<7 ; 0=\geq 7)\end{array}$ & 5,56 & $2,75-11,20$ & $<0,001$ \\
$\begin{array}{l}\text { Consultas pré-natal } \\
(1=\leq 6 ; 0=>6)\end{array}$ & 2,05 & $1,15-3,64$ & 0,014 \\
\hline
\end{tabular}

godness of fit test: Qui-quadrado Hosmer-Lemeshow $=5,28 ; \mathrm{p}=0,6253$

godness of fit test: chi-square Hosmer-Lemeshow $=5.28 ; p=0.6253$ 
talidade infantil neonatal, destacando-se a redução da mortalidade infantil pósneonatal como um dos principais determinantes desse elevação ${ }^{6,15,22,23}$.

A comparação dos resultados obtidos neste estudo com os de outros trabalhos que utilizaram a mesma abordagem metodológica revela que o sexo do nascido vivo não se associou à mortalidade infantil, ${ }^{13,15,19,24}$ porém diferindo de outros relatos da literatura ${ }^{25,26}$.

O baixo peso ao nascer, valores do índice de Apgar inferiores a sete no primeiro e quinto minutos de vida e a prematuridade apresentaram na coorte em estudo forte associação com a morte antes de completar o primeiro ano de vida, reforçando os estudos descritos na literatura de que são variáveis fortemente preditoras da mortalidade infantil ${ }^{8,13,15,19,24,25,27,28}$.

O baixo peso ao nascer é descrito como o fator de risco isolado que mais afeta as mortes antes do primeiro ano de vida ${ }^{13,24,25}$ e mencionado como um marcador do risco social relacionado-se às precárias condições socioeconômicas e ao comportamento materno em relação aos cuidados de saúde ${ }^{15,29}$.

A prematuridade tem sido referida como um dos fatores importantes da mortalidade perinatal e neonatal precoce, tendo a ruptura prematura das membranas, o trabalho de parto prematuro e a interrupção precoce da gestação em virtude de doença hipertensiva ou sangramentos vaginais como os principais mecanismos relacionados à etiologia da mesma ${ }^{13,15}$.

$O$ índice de Apgar reflete as condições de vitalidade do recém-nascido. A associação entre o índice de Apgar no primeiro e quinto minutos de vida e as mortes infantis encontradas nesse estudo foi descrita em estudo realizado na cidade do Recife $^{25}$, confirmando-se como uma variável preditora da mortalidade infantil. Em relação aos resultados obtidos nesse estudo, o índice de Apgar no quinto minuto de vida do recém-nascido obteve maior valor da medida de associação em relação ao índice de Apgar no primeiro minuto de vida.
Achado semelhante foi encontrado em estudo realizado no município de Montes Claros $-\mathrm{MG}^{24}$. O escore de Apgar no primeiro minuto de vida possui menor possibilidade teórica de indicar risco de morte do que o índice no quinto minuto ${ }^{30}$. Esses resultados sugerem a necessidade de medidas de suporte adequadas e continuadas, a fim de garantir maior sobrevida aos recém-nascidos com escore de Apgar inferior a sete no primeiro e quinto minutos de vida.

A associação entre as mortes infantis e a gravidez múltipla é conhecida e referida por vários autores ${ }^{13,15,20,25}$. Assim como nessa coorte de nascimentos, os municípios de Montes Claros - MG e Presidente Prudente - SP registraram coeficientes de mortalidade neonatal e infantil maiores entre os nascidos vivos oriundos de gestações múltiplas ${ }^{20,24}$. A análise multivariada dos estudos realizados em Goiânia, Montes Claros e Recife ${ }^{15,24,25}$, a exemplo desse estudo, também não demonstrou associação entre a gravidez múltipla e morte no período neonatal, devido provavelmente à atuação da idade gestacional e ao baixo peso ao nascer como fatores de confusão para a gemelaridade em relação ao risco de morte durante o período neonatal.

A freqüência às consultas de pré-natal tem sido demonstrada por alguns estudos como uma das variáveis mais importantes relacionadas à gestação e ao parto na prevenção da morbidade e mortalidade infantil e de seus componentes ${ }^{20,24,26,28}$. O acompanhamento rigoroso durante o pré-natal permite a identificação e intervenção precoces no sentido de minimizar danos à saúde materno-infantil. Sendo assim, a garantia de assistência pré-natal de qualidade adequadamente conduzido e a organização da assistência em sistemas hierarquizados e regionalizados de forma a garantir acessibilidade à gestante, pode detectar doenças maternas e fetais, melhorando assim a possibilidade de sobrevivência do recém-nascido e reduzindo a prevalência de retardo do crescimento intrauterino, a prematuridade e a ocorrência de 
baixo peso ao nascer ${ }^{20,23,24}$. O estudo de Menezes et al. (1998) identificou associação estatisticamente significante (RR ajustado $=3,09$ ) entre mães com menos de 5 consultas pré-natal e a ocorrência de óbito perinatal. Foi encontrada nesse estudo associação entre o número de consultas pré-natais e as mortes antes de completar o primeiro ano de vida, constatando-se RR não ajustado de 8,66 para nenhuma consulta pré-natal em relação a 6 consultas e mais. Definindo-se ponto de corte em menos de 4 consultas e de 4 e mais consultas pré- natal, o risco relativo não ajustado foi da ordem de 3,33 com IC $_{95 \%}[2,22-5,02] \mathrm{e}$ de 2,77 quando corte de menos de 7 consultas e de 7 e mais consultas pré-natal. Essa associação foi sustentada na análise multivariada desse estudo, com ponto de corte de 6 consultas de pré-natal.

A paridade materna, apesar de ser descrita como fator determinante para a morbimortalidade infantil e estar associada ao nível socioeconômico da família ${ }^{13}$, não apresentou nesse estudo diferenças significativas entre as primíparas e o grupo de multíparas, a exemplo dos resultados encontrados nos estudos desenvolvidos em Santo André e Pelotas ${ }^{13,19}$, que não encontraram associação entre o número de filhos tidos e os óbitos neonatais analisados. Entretanto, estudo sobre a mortalidade pós-neonatal realizado no Canadá29, com os dados referentes aos anos de 1978 e 1979, identificou associação estatisticamente significativa entre mortes no período pós-neonatal e nascimentos prévios, sugerindo o autor que a alta paridade pode estar relacionada a baixo nível socioeconômico.

O parto vaginal não esteve relacionado com a mortalidade infantil na coorte de nascimentos estudada. Comportou-se como variável de confusão no estudo realizado em Santo André, assumindo na análise bivariada efeito protetor em relação às mortes neonatais; no entanto, o fator de risco presente na associação foi o peso ao nascer após controle da variável tipo de parto $^{13}$. Não houve associação entre tipo de parto e mortes neonatais no estudo de uma coorte em Montes Claros e em Reci$\mathrm{fe}^{24,25}$, semelhante aos achados dessa coorte estudada e diferente dos resultados do estudo em Goiânia ${ }^{15}$, sobre o efeito protetor do parto cesariana, ressaltando sua maior ocorrência em hospitais privados daquele município, cuja população de melhor nível socioeconômico detém outras características importantes e determinantes da sobrevivência infantil.

A escolaridade da mãe é considerada como um marcador da condição socioeconômica da mãe e de sua família, relacionando-se nesse contexto com o perfil cultural e comportamental ligados aos cuidados de saúde, agindo como importante determinante das mortes infantis ${ }^{15,29}$. Na coorte de nascidos vivos em Maracanaú observou-se uma tendência de diminuição dos valores do risco relativo não ajustado à medida que o grau de escolaridade materna (em anos de estudo) aumentava, apesar da não ocorrência de associação estatisticamente significante na análise multivariada (permaneceu no modelo final como variável de controle) e percebeu-se que o coeficiente de mortalidade infantil foi maior entre nascimentos de mãe com nenhuma escolaridade em relação às demais categorias. Achado semelhante foi encontrado no estudo do município de Montes Claros - $\mathrm{MG}^{24}$, que descreve redução do coeficiente das mortes neonatais em relação ao aumento da escolaridade materna, porém sem associação estatisticamente significante. A coorte de nascimentos analisada no município de Santo André - $\mathrm{SP}^{13}$ também não verificou tendência de diminuição da probabilidade de morte com o aumento do grau de instrução da mãe. A análise de regressão logística do estudo de caso-controle desenvolvido em Recife - $\mathrm{PE}^{25}$ não encontrou associação das mortes neonatais e grau de instrução da mãe. No entanto, estudo realizado em Goiânia - $\mathrm{GO}^{15}$, por Morais Neto e Barros (2000), utilizando metodologia semelhante, encontrou associação estatística significante entre mortes no período pós- 
neonatal e mães sem instrução. Em conseqüência à exclusão de óbitos infantis do estudo, visto a não ligação com a base de dados de nascidos vivos, devemos considerar a possibilidade insuficiente de expressão da associação entre mortes infantis e grau de instrução materna. Cabe salientar que 4,4\% das DN analisadas não continham dados sobre a escolaridade materna, podendo ter contribuído para que essa variável não alcançasse associação com as mortes infantis estudadas.

A existência de maior probabilidade de morte infantil (neonatal e pós-neonatal) entre os nascidos vivos de mães com idades consideradas extremas, ou seja, com idade inferior a 20 anos e naquelas de 35 anos e mais, é sugerida por alguns autores $^{13,28}$. O resultado da análise bivariada obtida nesse estudo revelou associação estatisticamente significativa entre nascido vivos de mães com 35 anos e mais e a ocorrência de óbito infantil, porém esse resultado foi esvaziado na análise multivariada, diferente, portanto, dos achados do estudo em Caxias do Sul, cujo RR ajustado foi de 4,52 em relação aos nascimentos de mães com idade entre 20-34 $\operatorname{anos}^{28}$, e em Pelotas, cuja Odds Ratio ajustada manteve-se significante entre mães com idade igual ou superior a 35 anos $^{19}$. No entanto, o estudo de uma coorte de nascimentos da cidade de Montes Claros $^{24}$ e em Santo André ${ }^{13}$, o estudo de caso controle na cidade do Recife ${ }^{25}$ e o estudo realizado com os dados de nascidos vivos e óbitos em menores de um ano na cidade de Goiânia - $\mathrm{GO}^{15}$, não evidenciaram força de associação entre idade materna acima de 35 anos e óbito infantil e de seus componentes. Evidenciou-se risco para óbito no período pós-neonatal entre mães de nascidos vivos com menos de 25 anos de idade, tendo o risco aumentado entre mães com idade inferior a $20 \operatorname{anos}^{31}$. Várias explicações são dadas para o aumento das chances de óbito entre mães com idades extremas, dentre elas a ocorrência maior de patologias perinatais, maior possibilidade de imaturidade, aumento da proba- bilidade de baixo peso ao nascer e parto prematuro $^{9}$.

O local de nascimento pode constituir um fator de risco para as mortes infantis ${ }^{13}$. Esse estudo mostrou que os partos realizados em domicílio apresentaram maior chance de resultar em óbito infantil (RR não ajustado $\left.=5,92 ; \mathrm{IC}_{95 \%} 1,53-22,94\right)$ em relação aos partos ocorridos em hospitais, sugerindo que o atendimento pediátrico na sala de parto nos primeiros minutos de vida do recém-nascido parece ser vital para a diminuição da morbimortalidade, principalmente entre os neonatos, porém a associação não foi sustentada no modelo final da análise multivariada. O estudo realizado em Santo André também não encontrou associação estatisticamente significante entre nascimentos hospitalares e em outros locais (a caminho do hospital e em ambulâncias ou outros veículos) em relação aos óbitos ocorridos no período neonatal ${ }^{13}$.

Considerando que a categoria econômica dos estabelecimentos de saúde pode apresentar algum efeito sobre o risco de morte entre crianças menores de um ano de idade, principalmente entre os óbitos ocorridos no período neonatal, adotou-se nesse estudo as categorias de estabelecimento privado/conveniado com o SUS e público/atendimento exclusivo a clientela do SUS. Os resultados encontrados nessa coorte não sugerem associação entre mortes infantis e o tipo de hospital (público ou privado), diferem dos achados do estudo em Goiânia ${ }^{15}$, cujo risco de morte durante o período neonatal foi de 2,3 superior entre os nascimentos ocorridos em hospitais público-estatal em relação aos nascidos vivos em hospitais privados e de 2,7 maior durante o período pós-neonatal. Esses resultados remetem a discussão sobre o efeito protetor de nascimentos em hospitais privados, cuja população parece dispor de melhores condições socioeconômicas e, sendo assim, detendo outras características relevantes para a sobrevivência infantil.

Face aos resultados mais relevantes desse estudo, sugere-se o emprego da téc- 
nica de ligação de banco de dados por parte do nível municipal de atenção à saúde, tendo em vista seu baixo custo operacional e a possibilidade de planejamento e avaliação da atenção materno-infantil, permitindo a avaliação dos dados coletados, no sentido de alcançar dados cada vez mais confiáveis e a vigilância por parte dos serviços de saúde. Recomenda-se a vigilância por parte dos serviços de saúde aos recém-nascidos de baixo peso, prematuros e com escore de Apgar igual ou inferior a sete, no sentido de ofertar assistência adequada e tecnologicamente avançada para cada um dos níveis de complexidade do sistema local de saúde, bem como a garantia de assistência pré-natal de qualidade adequadamente conduzida e a organização da assistência em sistemas hierarquizados e regionalizados de forma a garantir acessibilidade à gestante, a fim de detectar precocemente eventuais doenças maternas e fetais, melhorando assim a possibilidade de sobrevivência do recémnascido e reduzindo a prevalência de retardo do crescimento intra-uterino, a prematuridade e a ocorrência de baixo peso ao nascer.

\section{Referências}

1. UNICEF. Fondo de las Naciones Unidas para la Infancia/ Oficina Regional para América Latina y el Caribe; CEPAL, Comissión Económica para América Latina y el Caribe/ Centro Latinoamericano de Demografia (CELADE). Mortalidade en la niñez - una base de dados desde 1960 Brasil. Chile: Santiago; 1993.

2. UNICEF. Fundo das Nações Unidas para a Infância. Situação Mundial da Infância. Brasília; 1989.

3. Barros FC, Victora CG. Epidemiologia da saúde infantilum manual para diagnósticos comunitários. HucitecUNICEF. São Paulo; 1994.

4. Barreto ICHC. Utilização de autópsias verbais na investigação da causa básica de óbito de crianças menores de um ano de idade em três municípios do Ceará [dissertação de mestrado]. Fortaleza: Faculdade de Medicina da Universidade Federal do Ceará; 1997.

5. Ribeiro VS, Silva AAM. Tendências da mortalidade neonatal em São Luís, Maranhão, Brasil, de 1979 - 1996. Cad Saúde Pública 2000; 16 (2): 429-38.

6. Carmo FLM. Fatores de risco para mortalidade neonatal em hospital público terciário no município de Fortaleza Ceará [dissertação de mestrado]. Fortaleza: Universidade Estadual do Ceará; 2002.

7. Carvalho HMB. Mortalidade neonatal evitável em hospital terciário do município de Fortaleza, Ceará [dissertação de mestrado]. Fortaleza: Universidade Estadual do Ceará; 2002.

8. Souza RKT, Gotlieb SLD. Probabilidade de morrer no primeiro ano de vida em área urbana da região Sul, Brasil. Rev Saúde Pública 1993; 27 (6): 445-54.

9. Oliveira LAP, Mendes MMS. Mortalidade infantil no Brasil: uma avaliação de tendências recentes. In: Minayo MCS. Os Muitos Brasis. São Paulo-Rio de Janeiro: Hucitec-Abrasco 1999. p. 291-301.
10. Oliveira H, Minayo MCS. A auto-organização da vida como pressuposto para a compreensão da morte infantil. Ciência e Saúde Coletiva 2001; 6: 139-49.

11. Almeida SDM, Barros MBA. Atenção à saúde e mortalidade neonatal: estudo caso-controle realizado em Campinas, SP. Rev Bras Epidemiol 2004; 7: 22-35.

12. Camargo Jr. KR, Coeli CM. Reclink: aplicativo para o relacionamento de bases de dados, implementando o método probabilistic record linkage. Cad Saúde Pública 2000; 16: 439-47.

13. Almeida MF. Mortalidade Neonatal em Santo André [tese doutorado]. São Paulo: Departamento de Epidemiologia da Faculdade de Saúde Pública da Universidade de São Paulo; 1994.

14. Almeida MF, Mello Jorge MHP. O uso da técnica de "linkage" de sistemas de informação em estudos de coorte sobre mortalidade neonatal. Rev Saúde Pública 1996; 30: 141-7.

15. Morais Neto OL, Barros MBA. Fatores de risco para mortalidade neonatal e pós-neonatal na Região Centro Oeste do Brasil: linkage entre bancos de dados de nascidos vivos e óbitos infantis. Cad Saúde Pública 2000; 16: 477-85.

16. Herrchen B, Gould JB, Nesbitt TS. Vital Statistics linked Birth / Infant Death and Hospital Discharge Record Linkage for Epidemiological Studies. Computers and Biomedical Research 1997; 30: 290-305.

17. Bohland AK, Mello Jorge MHP. Mortalidade infantil de menores de um ano de idade na região Sudoeste do Estado de São Paulo. Rev. Saúde Pública 1999; 33: 366 373. 
18. Theme Filha MM, Gama SGN, Cunha CB, Leal MC. Confiabilidade do Sistema de Informações sobre Nascidos Vivos Hospitalares no Município do Rio de Janeiro, 1999 - 2001. Cad. Saúde Pública 2004; 20 (S1): 583-91.

19. Menezes AMB, Victora CG, Barros FC, Albernaz E, Menezes FS, Jannke HÁ, Alves C, Rocha C. Mortalidade infantil em duas coortes de base populacional no Sul do Brasil: tendências e diferenciais. Cad Saúde Pública 1996; 12 (S1): 79-86.

20. Gomes JO, Santo AH. Mortalidade infantil em município da região Centro Oeste Paulista, Brasil, 1990 à 1992. Rev Saúde Pública 1997; 31:330-41.

21. Ribeiro VS, Silva AAM. Tendências da mortalidade neonatal em São Luís, Maranhão, Brasil, de 1979 - 1996. Cad Saúde Pública 2000; 16: 429-38.

22. Ahmad OB, Lopez AD, Inoue M. Reevaluación de la disminución de la mortalidad infantil. Bull World Health Organ 2000; 78: 1175-91.

23. Lansky S, França E, Leal MC. Mortalidade perinatal e evitabilidade: revisão da literatura. Rev Saúde Pública 2002; 36: 759-72.

24. Martins EF, Velásquez-Meléndez G. Determinantes da mortalidade neonatal a partir de uma coorte de nascidos vivos, Montes Claros, Minas Gerais, 1997 - 1999. Rev Bras Saúde Matern Infant 2004; 4: 405-12.
25. Sarinho SW, Filho DAM, Silva GAP, Lima MC. Fatores de risco para óbitos neonatais no Recife: um estudo casocontrole. Jornal de Pediatria 2001; 77: 294-8.

26. Menezes AMB, Barros FC, Victora CG, Tomasi E, Halpern R, Oliveira ALB. Fatores de risco para mortalidade perinatal em Pelotas, RS, 1993. Rev Saúde Pública 1998; 32: $209-16$

27. Barros FC, Victora CG, Tomasi E, Horta B, Menezes AM, Cesar JÁ, Halpern R, Olinto MT, Post CL, Costa JSD, Menezes FS, Garcia MM, Vaughan JP. Saúde maternoinfantil em Pelotas, Rio Grande do Sul, Brasil: principais conclusões da comparação dos estudos das coortes de 1992 e 1993. Cad Saúde Pública 1996; 12 (S1): 87-92.

28. Araújo BF, Bozzetti MC, Tanaka ACA. Mortalidade neonatal precoce no município de Caxias do Sul: um estudo de coorte. Jornal de Pediatria 2000; 76: 200-6.

29. Semenciw RM, Morrison HI, Lindsay J, Silins J, Sherman GJ, Mao Y, Wigle DT. Risk Factors for Postneonatal Mortality: Results from a Record Linkage Study. Int J Epidemiol 1986; 15: 369-72.

30. Coutinho SB. Mortalidade neonatal em cinco maternidades da cidade do Recife 1994: relatório de pesquisa. Recife: UNICEF; 1996.

Recebido em: 12/09/05 Versão reformulada reapresentada em: 02/03/06

Aprovado em: 15/03/06 